\title{
GENERALIZED MATRIX DETERMINANT LEMMA AND THE CONTROLLABILITY OF SINGLE INPUT CONTROL SYSTEMS
}

\author{
Robert Vrabel \\ Faculty of Materials Science and Technology \\ Slovak University of Technology in Bratislava \\ J. Bottu 25, 91701 Trnava, SLOVAKIA
}

\begin{abstract}
In this note, a recently derived generalization of the Matrix Determinant Lemma is used in the alternative proof of one of the fundamental results in modern control theory of the linear time-invariant systems $\dot{x}=A x+B u, y=C x$, namely that the state controllability is unaffected by state feedback, and even more specifically, that for the controllability matrices $\mathcal{C}$ of the single input open- and closed-loop systems the equality
\end{abstract}

$$
\operatorname{det}\left(\mathcal{C}_{(A, B, C)}\right)=\operatorname{det}\left(\mathcal{C}_{(A-B K, B, C)}\right)
$$

holds.

AMS Subject Classification: 93C05, 93B05, 15A24

Key Words: linear control system, generalized Matrix Determinant Lemma

\section{Introduction and Main Result}

Stability, controllability and observability are the important structural properties of dynamical systems and represent three major concepts of modern control system theory. With "stability," "controllability" and "observability," one can classify the control systems without first finding the solution in an explicit form. The last two concepts were introduced by R. E. Kalman in the early 60 s of the

$\begin{array}{lr}\text { Received: } & \text { June 8, } 2017 \\ \text { Revised: } & \text { July 3, 2017 } \\ \text { Published: } & \text { August 30, 2017 }\end{array}$

(c) 2017 Academic Publications, Ltd. url: www.acadpubl.eu 
last century, see e. g. [1]. The concept stability has a longer history, first mentioned in 1892 by A. M. Lyapunov in his Doctoral Dissertation [2]. As we will see later, these three concepts are closely related.

Let us consider a linear time-invariant (LTI) control system

$$
\dot{x}(t)=A x(t)+B u(t), y(t)=C x(t), x(0)=x_{0}
$$

The control $u(t)$ is a $m$-dimensional column vector function of time which must be chosen to make the system behave in a desired manner. $A, B$ and $C$ are $n \times n, n \times m$ and $q \times n$ constant matrices, respectively, and $x_{0} \in \mathbb{R}^{n}$ is an initial state of the system. In order to ensure clarity and consistency of presentation, let us define these three key concepts.

Stability. The LTI system, described by state equation in (1) is said to be (globally) asymptotically stable if the homogeneous response, that is, $u(t) \equiv 0$, of the state vector $x(t)$ returns to the origin $x=0$ of the state space from any initial state $x_{0}$ as time $t \rightarrow \infty$.

Controllability. The LTI system (1) or the triple $(A, B, C)$ is said to be state controllable if for any initial state $x(0)=x_{0}$ and any final state $x_{T}$, there exists a control input $\bar{u}(t)$ that transfers $x_{0}$ to $x_{T}$ in a finite time $T$.

Observability. The LTI system (1) or the triple $(A, B, C)$ is said to be observable if any initial state $x(0)$ can be uniquely determined from the knowledge of the output $y(t)$ and the input $u(t)$ on the interval $\left[0, t_{1}\right]$ for some $t_{1}>0$.

The simple algebraic conditions can be given for the asymptotic stability, controllability and observability of the LTI control system (1):

Theorem 1. A necessary and sufficient condition for $(A, B, C)$ to be asymptotically stable is that every eigenvalue of $A$ has a strictly negative real part.

Theorem 2 (Kalman). A necessary and sufficient condition for $(A, B, C)$ to be controllable is

$$
\operatorname{rank} \mathcal{C}_{(A, B, C)}=\operatorname{rank}\left[B \vdots A B \vdots A^{2} B \vdots \cdots \vdots A^{n-1} B\right]=n .
$$

Theorem 3 (Kalman). A necessary and sufficient condition for $(A, B, C)$ to be observable is

$$
\operatorname{rank} \mathcal{O}_{(A, B, C)}=\operatorname{rank}\left[C^{T} \vdots A^{T} C^{T} \vdots\left(A^{T}\right)^{2} C^{T} \vdots \ldots \vdots\left(A^{T}\right)^{n-1} C^{T}\right]^{T}=n .
$$

$A$ superscript $T$ denotes the matrix transpose operation.

Consider the state feedback control $u(t)=-K x(t)+r(t)$, where $K$ is an $m \times$ $n$ gain matrix and $r(t)$ is an $m$-dimensional external input. Then a closed-loop 
system dynamics is given by $\dot{x}(t)=(A-B K) x(t)+B r(t)$. A fundamental result of the linear control theory is that the five following conditions are equivalent:

(i) the triple $(A, B, C)$ is controllable;

(ii) the triple $\left(A^{T}, C^{T}, B^{T}\right)$ is observable;

(iii) $\operatorname{rank} \mathcal{C}_{(A, B, C)}=n$;

(iv) $\operatorname{rank}\left[A-\lambda I_{n} \vdots B\right]=n$, for every $\lambda \in \mathbb{C}$;

(v) for every $\alpha_{0}, \alpha_{1}, \ldots, \alpha_{n-1} \in \mathbb{R}$ there exists a matrix $K \in \mathbb{R}^{m \times n}$ such that $\chi_{A-B K}(\lambda)=\lambda^{n}+\alpha_{n-1} \lambda^{n-1}+\cdots+\alpha_{1} \lambda+\alpha_{0}$, where $\chi_{A-B K}$ is the characteristic polynomial of the matrix $A-B K$.

Another important property is the fact that controllability is unaffected by state feedback.

Theorem 4. The triple $(A, B, C)$ is controllable if and only if the triple $(A-B K, B, C)$ is controllable for all $K$ of dimension $m \times n$.

PROOF. The statement follows from the Belovich-Popov-Hautus test (the point (iv)) and the matrix identity

$$
\left[(A-B K)-\lambda I_{n} \vdots B\right]=\left[A-\lambda I_{n} \vdots B\right]\left[\begin{array}{rr}
I_{n} & 0 \\
-K & I_{n}
\end{array}\right] .
$$

In this note we prove the stronger statement of Theorem 4 for the case when the controllability matrices $\mathcal{C}_{(A, B, C)}$ and $\mathcal{C}_{(A-B K, B, C)}$ are the square matrices, namely

Theorem 5. For $m=1$ and $n \geq 2$ is

$$
\operatorname{det}\left(\mathcal{C}_{(A, B, C)}\right)=\operatorname{det}\left(\mathcal{C}_{(A-B K, B, C)}\right)
$$

\section{Proof of Theorem 5}

We precede the proof of Theorem 5 by the lemma which is a generalization of the well-known Matrix Determinant Lemma (MDL) representing an important analytical tool in the matrix theory. For the wider context of MDL in the linear algebra see e. g. [3].

Lemma 6. (Generalized MDL, [4]) Suppose $H$ is a square matrix of dimension $n$ and $u_{i}, v_{i}$ are the $n \times 1$ column vectors, $i=1, \ldots, k$. Then for every $k \geq 1$ we have the equality

$$
\operatorname{det}\left(H+\Delta_{k}\right)=\operatorname{det}(H)+\sum_{i=1}^{k} v_{i}^{T} \operatorname{adj}\left(H+\Delta_{i-1}\right) u_{i},
$$


where

$$
\Delta_{i}= \begin{cases}n \times n \text { zero matrix } & \text { for } i=0, \\ \sum_{j=1}^{i} u_{j} v_{j}^{T} & \text { for } i=1, \ldots, k .\end{cases}
$$

Proof of Theorem 5: Let us denote the column vectors $(A-B K)^{i} B-A^{i} B$ by $\beta_{i}, i=1, \ldots, n-1$. Now we rewrite $\mathcal{C}_{(A-B K, B, C)}$ as the following sum:

$$
\begin{aligned}
& \mathcal{C}_{(A-B K, B, C)}=\left[B \vdots(A-B K) B \vdots(A-B K)^{2} B \vdots \cdots \vdots(A-B K)^{n-1} B\right]
\end{aligned}
$$

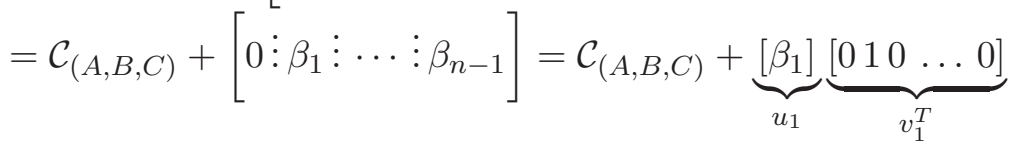

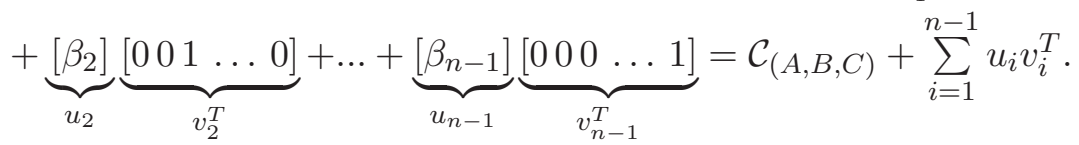

Then on the basis of Lemma 6 for $H=\mathcal{C}_{(A, B, C)}$ and $k=n-1$ we obtain the equality

$$
\operatorname{det}\left(\mathcal{C}_{(A-B K, B, C)}\right)=\operatorname{det}\left(\mathcal{C}_{(A, B, C)}\right)+\sum_{i=1}^{n-1} v_{i}^{T} \operatorname{adj}\left(\mathcal{C}_{(A, B, C)}+\Delta_{i-1}\right) u_{i}
$$

In the iterative way we show that $v_{i}^{T} \operatorname{adj}\left(\mathcal{C}_{(A, B, C)}+\Delta_{i-1}\right) u_{i}=0$ for all $i=1, \ldots, n-1$ :

$\mathbf{i}=1: v_{1}^{T} \operatorname{adj}\left(\mathcal{C}_{(A, B, C)}\right) u_{1}=\operatorname{det}\left(H_{1}\right)$, where $H_{1}$ is the matrix obtained from $\mathcal{C}_{(A, B, C)}$ by replacing the second column of $\mathcal{C}_{(A, B, C)}$ by the column $\beta_{1}$;

$\mathbf{i}=\mathbf{2}: v_{2}^{T} \operatorname{adj}\left(\mathcal{C}_{(A, B, C)}+u_{1} v_{1}^{T}\right) u_{2}=\operatorname{det}\left(H_{2}\right)$, where $H_{2}$ is the matrix obtained from $H_{1}$ by replacing the third column of $H_{1}$ by the column $\beta_{2}$;

...

$\mathbf{i}=\mathbf{n}-\mathbf{1}: v_{n-1}^{T} \operatorname{adj}\left(\mathcal{C}_{(A, B, C)}+\Delta_{n-2}\right) u_{n-1}=\operatorname{det}\left(H_{n-1}\right)$, where $H_{n-1}$ is the matrix obtained from $H_{n-2}$ by replacing the $n$-th column of $H_{n-2}$ by the column $\beta_{n-1}$.

Because

$$
[B]\left(=1^{\text {st }} \text { column in } H_{i}\right)(-K B)=-B K B=\left[\beta_{1}\right]\left(=2^{\text {nd }} \text { column in } H_{i}\right),
$$

the determinant of $H_{i}$ is equal to zero for all $i=1, \ldots, n-1$. This completes the proof of Theorem 5 .

\section{Acknowledgements}

This publication is the result of implementation of the project "Increase of Power Safety of the Slovak Republic" (ITMS: 26220220077) supported by the 
Research \& Development Operational Program funded by ERDF.

\section{References}

[1] R. E. Kalman, Mathematical description of linear dynamical systems, Journal of the Society for Industrial \&5 Applied Mathematics, Ser. A, Control, 1 (1963), 152-192.

[2] A. M. Lyapunov, The General Problem of the Stability of Motion (In Russian), Doctoral dissertation, Univ. Kharkov (1892). English translations: The General Problem of the Stability of Motion, (A. T. Fuller trans.) Taylor \& Francis, London (1992).

[3] D. A. Harville, Matrix Algebra From a Statistician's Perspective, Springer-Verlag, New York (2008), doi: 10.1007/b98818.

[4] R. Vrabel, A note on the Matrix Determinant Lemma, International Journal of Pure and Applied Mathematics, 111, No. 4 (2016), 643-646, doi: 10.12732/ijpam.v111i4.11. 
